\title{
Cost-competitive Steel Devices for Seismic Retrofitting of RC Frames: Model Identification and Nonlinear Analysis
}

\section{Carmine Lima, Enzo Martinelli* and Ciro Faella}

Department of Civil Engineering, Via Giovanni Paolo II, University of Salerno, Fisciano (SA), Italy

\begin{abstract}
Seismic retrofitting of existing reinforced concrete (RC) buildings, designed in the last decades in seismic areas, is one of the most complex tasks for structural engineers: in fact, it includes several issues, such as quantifying the capacity of existing members, designing the supplemental ones and analysing the whole structure.

This paper is intended as a contribution to clarifying some of those issues. First of all, a model based on using 1D finite elements with fiber section discretization is proposed for simulating the behaviour of a cost-competitive steel device that can be employed as a link in Y-shaped eccentric bracings (EB): particularly, the cyclic response and the low-cycle fatigue degradation is modelled, based on the results of obtained in a previous experimental research carried out at the University of Salerno. Secondly, the global response of an existing RC frame equipped with the aforementioned devices is investigated via Non Linear Time History (NLTH) analyses. Taking into account the lowcycle fatigue often leads to significantly more severe seismic displacement demand value on the retrofitted structure: a close correlation is unveiled between some specific features of the seismic signals adopted in the NLTH and the actual influence of low-cycle fatigue.
\end{abstract}

Keywords: Steel braces; Low-cycle fatigue; Seismic retrofitting; Steel slit shape devices; Nonlinear analysis

\section{Introduction}

Reinforced Concrete (RC) structures and buildings designed and realised in the past decades in earthquake-prone zones are often characterised by significant levels of vulnerability, as highlighted by the damage and collapses observed in recent seismic events [1]. Therefore, existing RC buildings are generally in need for retrofitting in order to enhance their level of seismic safety according to the design codes currently in force [2].

In principle, several retrofitting strategies can be pursued. Some of them are based on adding further structural systems, such as bracings, which are often made of steel. Moreover, these structural systems include components that are capable of dissipating the input seismic energy [3]. Although several physical phenomena (such as friction of sliding surfaces, viscosity of fluids, yielding of metals, and so on) are considered for designing and realising these dissipative components, devices based on the hysteretic behaviour induced by the cyclic response of steel elements deformed beyond their yielding limit are the most frequently employed ones [4]. Consequently, the various shapes currently available on the market are related to the different physical phenomena resulting in hysteretic dissipation. In fact, these elements can yield under axial forces (i.e., Buckling Restrained Bracings) [5], bending moments (i.e., ADAS, TADAS, "long" links, etc.) [6-10], shear (i.e., "short" links, shear panels) and torsion $[11,12]$.

Moreover, several cost-competitive seismic devices can be specifically designed and obtained from commercial steel profiles through conventional steel work procedure $[8,9]$. One of these devices, often referred to as "Steel Slit Shape" (SSS) devices, can be obtained by carving a simple I-wide flange section profile stub in order to turn the web panel into a batch of steel stripes characterised by a low yielding limit and, hence, prone to exhibit a hysteretic response under cyclic actions with reduced exposures to local buckling phenomena, but a pronounced progressive degradation of the stiffness and strength [13]. Therefore, these devices can be employed as dissipative devices in Y-shape eccentric braces (EB) and ideally employed for seismic retrofitting of RC frames [14].

This paper is intended at investigating the possibility of employing SSS devices in steel bracings for retrofitting ordinary structures, where budget restrictions are often the key constraints. Some steel slit devices were obtained by carving a steel plate (i.e. the web of a I-wide flange section stub) in order to find a batch of steel stripes characterised by a low yielding limit and, then, prone to exhibit an hysteretic response under cyclic actions. They were tested at the Laboratory of Materials testing and Structures (LMS) of the University of Salerno [15]. Although these solutions have been already investigated by the scientific community to understand the key aspects of their mechanical behaviour and, then, their suitability as a possible solution in seismic design of steel bracing systems, the present study is intended to provide engineers with a deeper understanding of their performance under cyclic actions. The experimental results demonstrated that the strength degradation achieved under moderate cyclic actions expected in medium-to-high seismic risk zones slightly affects the failure modes of these devices. Then, the obtained results were used in deriving the low-cycle fatigue curve [15], which represents a general conceptual tool capable to describe the displacement capacity and quantify its progressive reduction due to the damage accumulation induced by cyclic actions beyond the actual yielding limit of the devices under consideration.

*Corresponding author: Enzo Martinelli, Department of Civil Engineering, Via Giovanni Paolo II, University of Salerno, Fisciano (SA), Italy, Tel: 39089 961111; E-mail: e.martinelli@unisa.it

Received October 23, 2015; Accepted November 24, 2015; Published November 30, 2015

Citation: Lima C, Martinelli E, Faella C (2015) Cost-competitive Steel Devices for Seismic Retrofitting of RC Frames: Model Identification and Nonlinear Analysis. J Steel Struct Constr 1: 104. doi:10.4172/2472-0437.1000104

Copyright: ( 2015 Lima C, et al. This is an open-access article distributed under the terms of the Creative Commons Attribution License, which permits unrestricted use, distribution, and reproduction in any medium, provided the original author and source are credited. 
In this paper the aforementioned "Steel Slit" devices are modelled in OpenSEES [16] by using only 1D Finite Elements including the low-cycle fatigue effects. First of all, the parameters employed for simulating the hysteretic response and the low-cycle fatigue effects are carefully identified for reproducing the behaviour observed on the tested devices [17]. Then, Incremental Dynamic Analyses (IDA) of a RC frame strengthened by Y-shape eccentric bracings whose short link is realised by the steel slit devices under consideration are performed for scaling the member-level behaviour observed in these experiments up to the global seismic response of the aforementioned RC frame. These analyses are mainly aimed at investigating the consequences of low-cycle fatigue affecting the dissipative devices on the global seismic response of the structure under consideration. The results obtained are finally related to the characteristics of the considered seismic signals, such as Peak Ground Acceleration (PGA) [18], Peak Ground Velocity $(P G V)$ and Damage Index $\left(I_{D}\right)[19]$. As a matter of fact, a close correlation is unveiled between some specific features of the seismic signals and the actual influence of the aforementioned phenomenon on the response of the strengthened RC frame under consideration.

\section{Overview of the experimental tests and results}

The Steel Slit Shape (SSS) considered in this paper were tested as part of a wide experimental campaign including other devices, such as short link and torsional members, which are not considered herein [15]. Particularly, the results obtained in the tests carried out on six SSS hysteretic devices (three of them made of S355 steel and other three ones made of S275 steel type [20]) with equal geometric dimensions are taken into account in the present study. They were designed according to the analytical relationship provided by Chan [21]in order to have the same values of yielding displacement $\delta_{E}$ and elastic stiffness $k_{E}$. The geometric dimensions of the device (and the slits) realised by conventional steelwork procedures are shown in Figure 1. The slotted holes had $20 \mathrm{~mm}$ diameter for a length of $205 \mathrm{~mm}$, in order to have 4 strips with the following dimensions: width $=55 \mathrm{~mm}$; thickness $=10$ $\mathrm{mm}$; depth of the part with constant cross-section $=185 \mathrm{~mm}$ (Figure 1 ).

All tests were performed in displacement control. Both constant and incremental amplitude cyclic tests were performed [22]. In particular, the amplitudes of constant amplitude cyclic tests were defined as a function of the displacement $\delta_{U}$ corresponding to the minimum nominal rotational capacity defined for short links by the Italian Code [20] (namely, $0.08 \mathrm{rad}$ ). Particularly, two tests were carried out by assuming a constant amplitude just equal to $\delta_{U}$, while other two were conducted with an amplitude of about $50 \%$ of $\delta_{U^{*}}$. Table 1 summarises the key information about the loading protocol and results of the six specimens.

The cyclic response exhibited by the SSS devices was initially characterised by wide hysteretic cycles, albeit affected by a significantly fast progressive decay in strength after few cycles (Table 1).

Figure 2 shows the cyclic response observed in the tests referred to specimens made of S355 steel. It shows the sudden strength decay observed in the test on the specimen SSS01-S355 after the third cycle (Figure 2a), as already reported in Table 2. Moreover, it shows the more gradual reduction in strength observed for the specimen SSS02-S355 (Figure 2b), subjected to a smaller displacement. The specimen SSS03-S355 (Figure 2c), tested under the incremental loading protocol, shows the regular shapes of the first cycles, characterised by the lower amplitudes, and the progressive strength decay resulting in a rather quick failure of the specimen.

Such a failure condition was actually achieved for a displacement amplitude of about $20 \mathrm{~mm}$ and, then, significantly lower the maximum one $\left(\delta_{U}=28 \mathrm{~mm}\right)$. This clearly emphasises a low-cycle fatigue phenomenon affecting the response of the tested specimens depending on the actual cyclic load history.

Similar considerations can be derived by observing the curves reported in Figure 3, ideally corresponding to Figure 2, but related to the SSS specimens made with S275 steel. The lower yielding stress (and strain) of SSS04 specimen (Figure 3a), with respect of SSS01, led to an even more abrupt shear force decay and a failure condition achieved

\begin{tabular}{|c|c|c|c|c|c|c|}
\hline \multirow[t]{2}{*}{ Steel type } & \multirow[t]{2}{*}{ Sample } & \multirow[t]{2}{*}{$\begin{array}{l}\text { Test amplitude } \\
(\mathrm{mm})\end{array}$} & \multicolumn{2}{|c|}{$\begin{array}{l}\text { Maximum } \\
\text { strength }\end{array}$} & \multicolumn{2}{|c|}{$\begin{array}{c}\text { Failure }(\cong 20 \% \\
\text { decay in strength) }\end{array}$} \\
\hline & & & Cycle & $\begin{array}{l}F_{\max } \\
{[\mathrm{kN}]}\end{array}$ & Cycle & $\begin{array}{c}\mathbf{F}_{\mathbf{u}} \\
{[\mathbf{k N}]}\end{array}$ \\
\hline \multirow[t]{4}{*}{ S355 } & SSS01 & 28.0 & 1 & 206.3 & 3 & 167.9 \\
\hline & SSS02 & 16.0 & 4 & 185.5 & 11 & 169.6 \\
\hline & SSS03 & incremental & 14 & 207.0 & 15 & 196.2 \\
\hline & SSS04 & 28.0 & 1 & 174.2 & 2 & 154.0 \\
\hline \multirow[t]{2}{*}{ S275 } & SSS05 & 16.0 & 3 & 159.5 & 6 & 127.8 \\
\hline & SSS06 & incremental & 13 & 163.4 & 14 & 147.8 \\
\hline
\end{tabular}
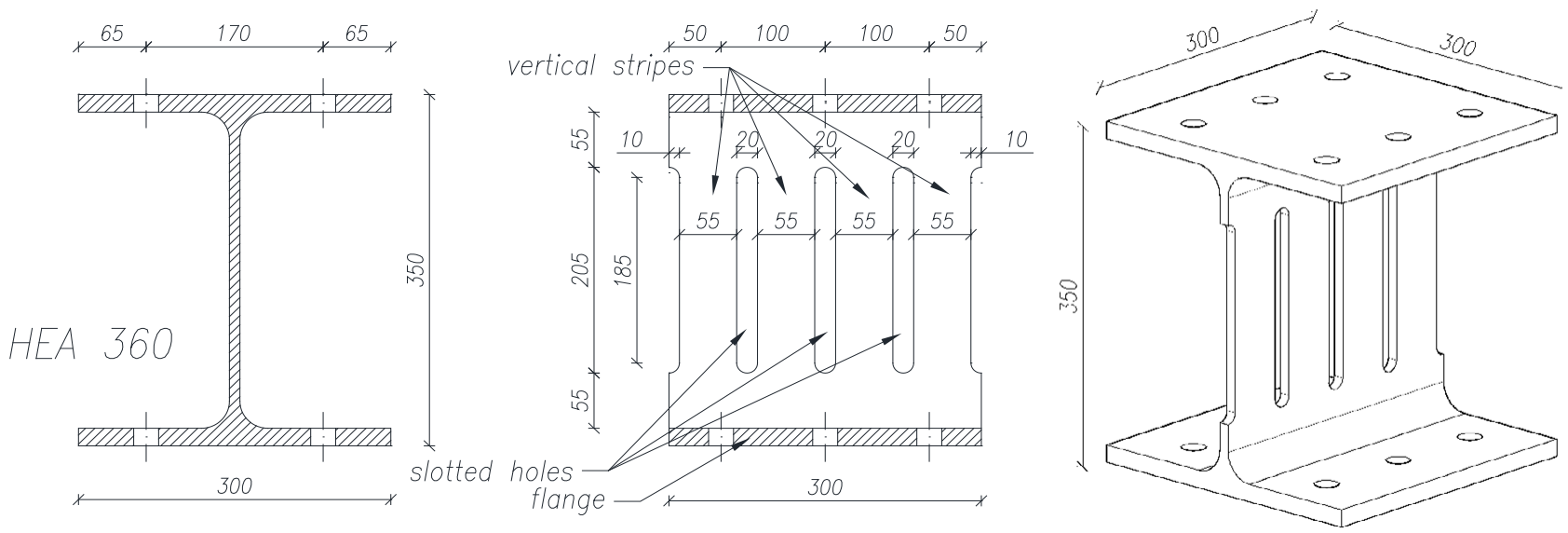

Figure 1: Geometric properties of the tested devices. 


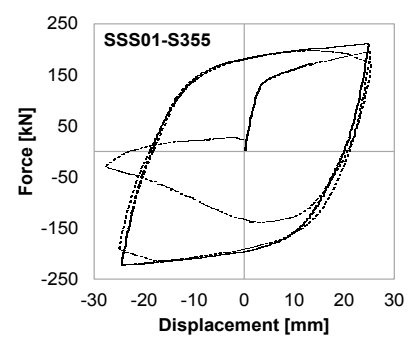

(a)

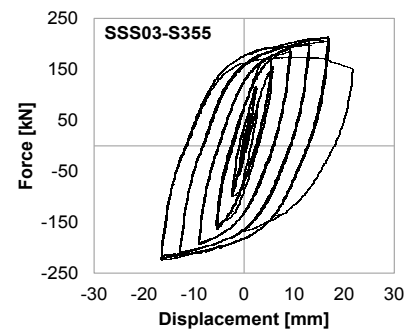

(c)

Figure 2: Cyclic response of tests SSS01 (a), SSS02 (b) and SSS03 (c).

\begin{tabular}{|c|c|c|c|c|c|c|}
\hline $\begin{array}{l}\text { SSS } \\
\text { Material }\end{array}$ & \multicolumn{6}{|c|}{ Opensees material } \\
\hline \multirow{3}{*}{ S355 } & \multirow{2}{*}{ Steel02 } & $\$ F y=355 \cdot 1,30$ & $\$ E=210000^{*} 0,3$ & $\$ b=0,01$ & $\$ R 0=15$ & $\$ c R 1=0,925$ \\
\hline & & $\$ c R 2=0,15$ & $\$ a 1=0,10$ & $\$ \mathrm{a} 2=1,50$ & $\$ a 3=0,10$ & $\$ a 4=1,50$ \\
\hline & Fatigue & $\$ E 0=0,37279$ & $\$ m=-0,415$ & & & \\
\hline \multirow{3}{*}{ S275 } & \multirow{2}{*}{ Steel02 } & $\$ F y=275 \cdot 1,30$ & $\$ E=210000 * 0,3$ & $\$ b=0,01$ & $\$ R 0=15$ & $\$ c R 1=0,925$ \\
\hline & & $\$ c R 2=0,15$ & $\$ a 1=0,10$ & $\$ \mathrm{a} 2=1,50$ & $\$ a 3=0,10$ & $\$ a 4=1,50$ \\
\hline & Fatigue & $\$ E 0=0,48125$ & $\$ m=-0,415$ & & & \\
\hline
\end{tabular}

Table 2: Numerical values adopted in Steel02 and Fatigue materials.

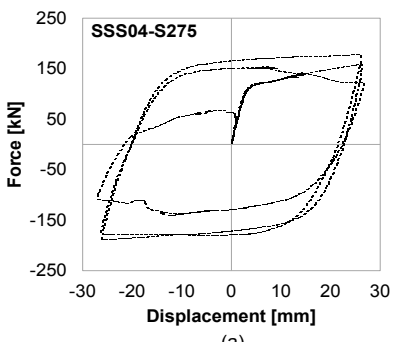

(a)

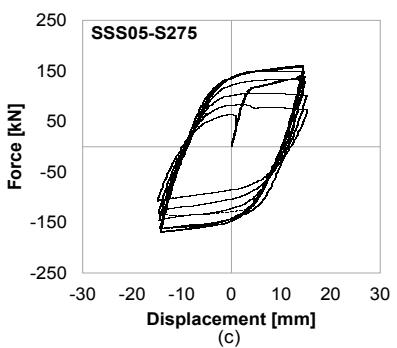

Figure 3: Cyclic response of tests SSS04 (a), SSS05 (b) and SSS06 (c).

at the end of the second cycle. Moreover, Figures $3 \mathrm{~b}$ and $3 \mathrm{c}$ shows the more progressive damage propagation occurring in the specimen SSS06, tested under an incremental cyclic load protocol.

As a matter of fact, the strength decay observed in all specimens was due to the occurrence of cracks developing at the round fillet of the steel stripe where significant localisation of the inelastic strains was observed (Figure 4).

\section{Calibration of the low-cycle fatigue curve}

The experimental tests outlined in Section 2 on the hysteretic devices demonstrated that they comply with the basic ductility requirements fixed by the European [1] and the Italian [20] provisions for steel components to be possibly employed as short links in eccentric steel bracing systems. Particularly, the constant amplitude cyclic test demonstrated that SSS devices under consideration were capable to comply reach the displacement $\delta_{U}$ corresponding to the minimum rotation capacity.

Therefore, a more comprehensive definition of the actual displacement/rotation capacity should be adopted to describe the cyclic performance of dissipative steel devices.

As observed in experimental tests, fatigue damage increases with applied cycles in a cumulative manner which may lead to fracture. This phenomenon can be simulated through to the "linear rule" suggested by Palmgren [23] and expressed in a mathematical form by Miner [24].

Then, the experimental results were employed to calibrate the low-cycle fatigue curve in the framework of the Miner's rule [24] relating the actual displacement capacity (in terms of maximum plastic displacement $\Delta_{p}$ under an ideally constant amplitude cyclic protocol) and the corresponding number of cycles $\mathrm{N}$ after which the device is expected to fail [15]:

$$
\Delta_{\mathrm{p}}=\mathrm{a} \cdot(2 \mathrm{~N})^{\mathrm{b}},
$$

in which $2 \mathrm{~N}$ is number of cycle reversals, while $a$ and $b$ are two coefficients that should be calibrated on experimental results.

Both constant and incremental amplitude protocols have been considered in the experimental tests proposed in this study. On the one hand, the former have been directly utilised to obtain one point for each test in the ideal $2 N-\Delta_{p}$ plane. On the other hand, the latter have also been utilised to calibrate the two coefficients of equation (1) by solving it with respect to $N$ introducing the resulting expression for each displacement amplitude $\Delta_{p, i}$ within the following equation deriving directly by the Miner's rule [24]:

$$
\sum_{\mathrm{i}=1}^{\mathrm{k}} \frac{\mathrm{n}_{\mathrm{i}}}{\mathrm{N}\left(\Delta_{\mathrm{p}, \mathrm{i}} ; \mathrm{a}, \mathrm{b}\right)}=1,
$$

where $k$ is the number of equal-amplitude cycle groups imposed in the experimental tests, $n_{i}$ is the number of cycles for each group, $\Delta_{p, i}$ their amplitude and $a$ and $b$ the two (unknown) coefficients introduced in equation. (1).
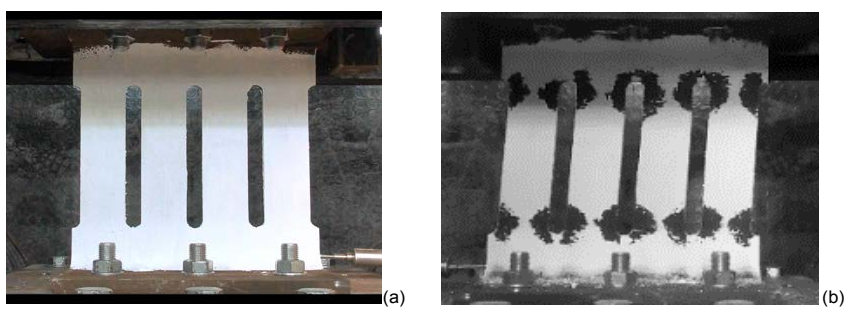

Figure 4: SSS device before (a) and at the end (b) of the cyclic test. 
Thus, the results of the six experimental tests reported in Section 2 have been employed to determine the two values $(a, b)$ which characterise the low-cycle fatigue curve for the devices considered in this study. Since both S355 and S275 devices exhibited similar performances, a unique couple of coefficients $a$ and $b$ was assumed for the fatigue curve (equation. (1)). Such a curve is plotted in Figure 5 along with the numerical values of the coefficients $a$ and $b$. In particular $a=56.264$ and $b=-0.415$ were calibrated on the experimental results.

It is worth highlighting that a unique curve describes the cyclic decay of the mechanical properties of all SSS devices analysed in this study.

\section{FE modelling of the STEEl Slit devices}

An accurate numerical model was implemented and calibrated in OpenSEES [16]. The four vertical stripes of the Steel Slit device were modelled with distributed plasticity elements by means of 100 fibers, while the flanges at the bottom and top were simulated as infinitely elastic elements connecting the ends of the stripes (Figure 6).

According to the overstrength observed in tensile tests on the materials, yielding stresses equal to $460 \mathrm{MPa}$ and $357 \mathrm{MPa}$ were used for steel S355 and S275, respectively. The nonlinear mechanical behaviour of steel was considered by adopting the "Steel02" uniaxial material law.

As suggested by Chan and Albermani [25], both longitudinal and tangential elastic moduli were multiplied by a 0.3 factor in order to reduce elastic moduli and simulating indirectly the significant flexibility affecting the node connecting the stripes and the elastic flange. The effects of the low-cycle fatigue were included in the model through the "Fatigue" material model taking into account a modified

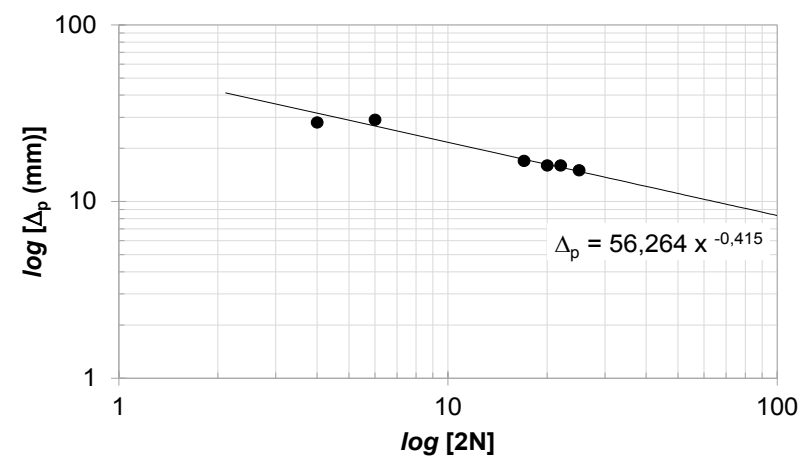

Figure 5: Low-cycle fatigue curve for SSS devices. rainflow counter and a linear strain accumulation model, based on Coffin-Manson log-log plot describing low cycle fatigue failure [26]. The deformation amplitude at each cycle is monitored and a cumulative damage of the steel fiber under consideration is defined. The fatigue law works in series with the "Steel02" material without affecting its stressstrain relationship until the damage function achieve the unit value. Conversely, once the "Fatigue" material model reaches a damage level of 1.0, the stress of the "Steel02" material becomes zero as a result of the low-cycle fatigue failure of the steel fiber.

Two values were calibrated for modelling the "fatigue" material of OpenSEES: the ultimate strain under monotonic action and the slope of the curve in log-log space (namely, $E_{0}$ and $m$, respectively, as defined within the OpenSEES command language manual [16]). On the one hand, the latter was directly derived from the experimental low-cycle fatigue curve and, hence, $\mathrm{m}=\mathrm{b}=-0.415$; on the other hand, the parameter $E_{0}$ was derived as follow from the value $\mathrm{a}=56.264$ of the same curve:

$$
\mathrm{E}_{0}=\frac{\mathrm{a}}{\mathrm{Q}_{\mathrm{pl}, \mathrm{Sss}}}
$$

where

$$
\mathrm{Q}_{\mathrm{pl}, \mathrm{SSS}}=\frac{2 \cdot \mathrm{M}_{\mathrm{pl}, \mathrm{SSS}} \cdot \mathrm{n}_{\mathrm{s}}}{\mathrm{H}}=\mathrm{n}_{\mathrm{s}} \cdot \frac{\mathrm{t}_{\mathrm{w}} \cdot \mathrm{B}^{2}}{2 \cdot \mathrm{H}} \cdot \gamma_{\mathrm{os}} \cdot \mathrm{f}_{\mathrm{y}}
$$

in which $Q_{p l, S S S}$ is the horizontal force associated to the plastic moment $M_{p l s s s}, \gamma_{o s}$ is the overstrength factor of steel assumed equal to 1,30 according to experimental tensile tests and $n_{s}$ indicates the number of stripes in the device.

Table 2 reports the numerical values adopted for the Steel02 and Fatigue material for simulating the Steel Slit devices made of S355 and S275 steel type, respectively. For the meaning of symbols refers to OpenSEES Command Language Manual [16].

Reversed cyclic pushover analyses were performed in displacement control on the FE models of the devices with the aim of reproducing the loading history observed in the experimental tests. The results shown in Figures 7 and 8, which compare the cyclic force-displacement response (in grey) and the experimental ones (in black), demonstrate that the model is capable of reproducing strength, stiffness and cyclic damage related to the low-cycle fatigue phenomena observed in the experimental campaign.

\section{Seismic response of an existing $\mathrm{RC}$ frame equipped with steel slit devices}

An existing four-storey frame structure, obtained through a simulated design procedure carried out for only gravitational loads

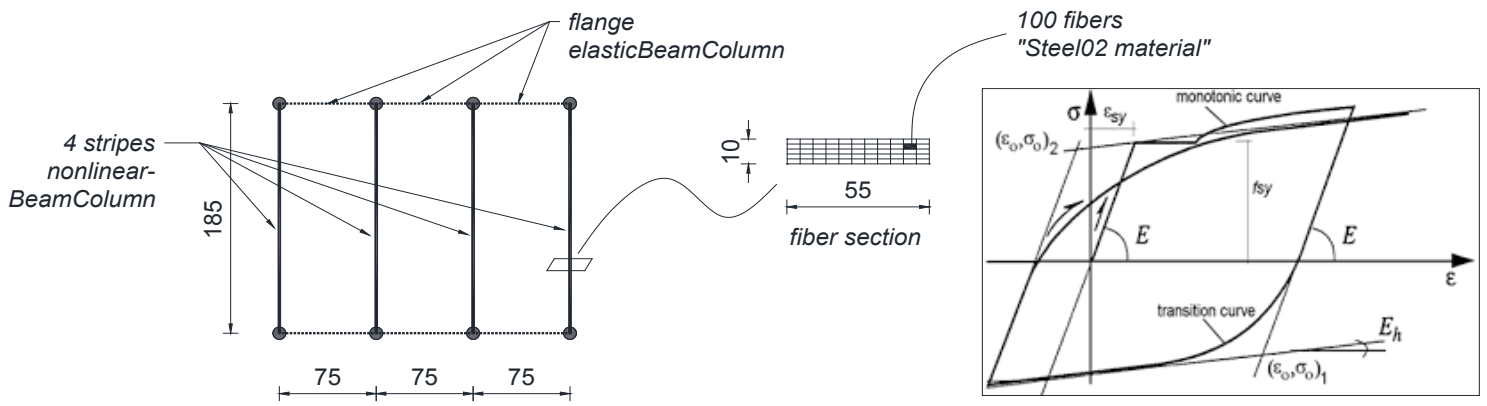

Figure 6: Steel Slit device modelling in Open SEES. 
Citation: Lima C, Martinelli E, Faella C (2015) Cost-competitive Steel Devices for Seismic Retrofitting of RC Frames: Model Identification and Nonlinear Analysis. J Steel Struct Constr 1: 104. doi:10.4172/2472-0437.1000104
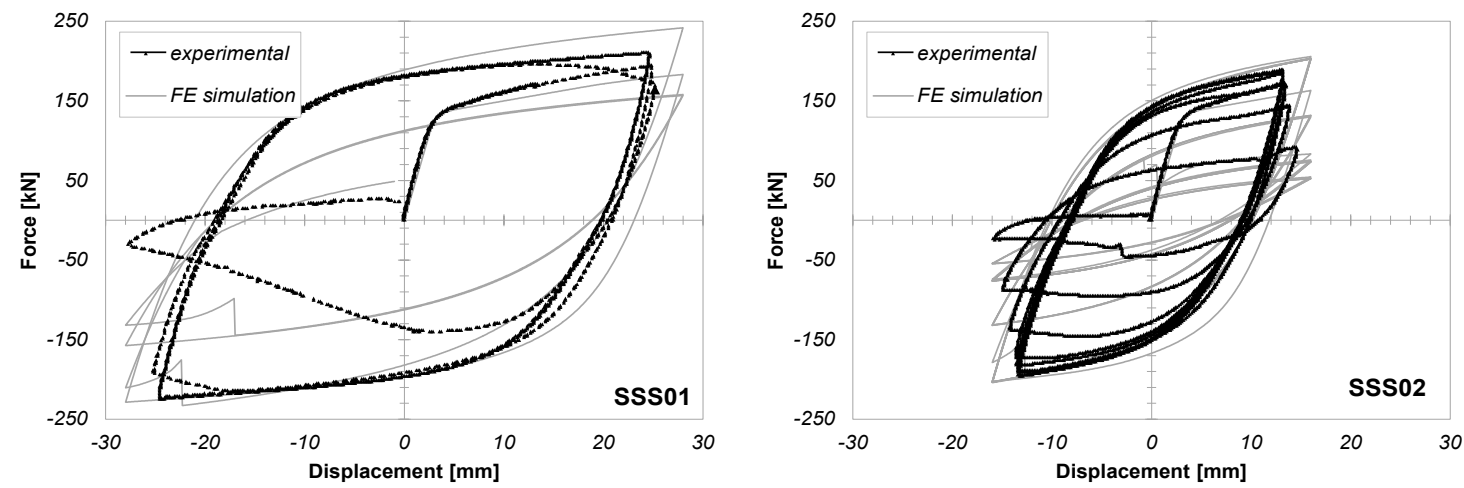

Figure 7: Comparison of the analytical and experimental cyclic response of SSS devices S355 steel type.
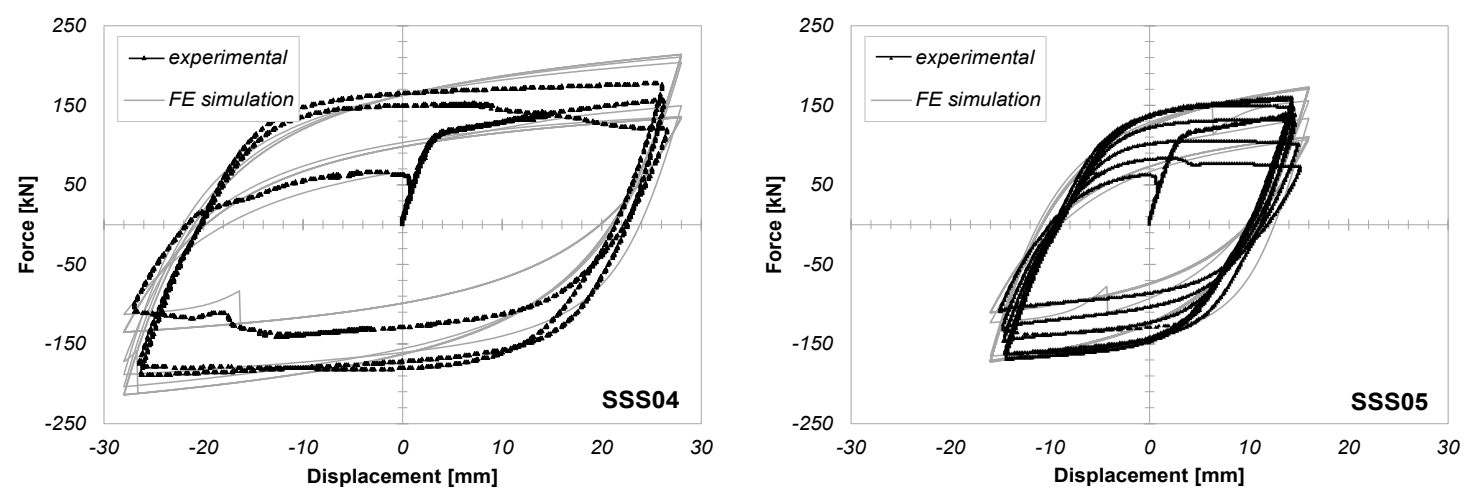

Figure 8: Comparison of the analytical and experimental cyclic response of SSS devices S275 steel type.

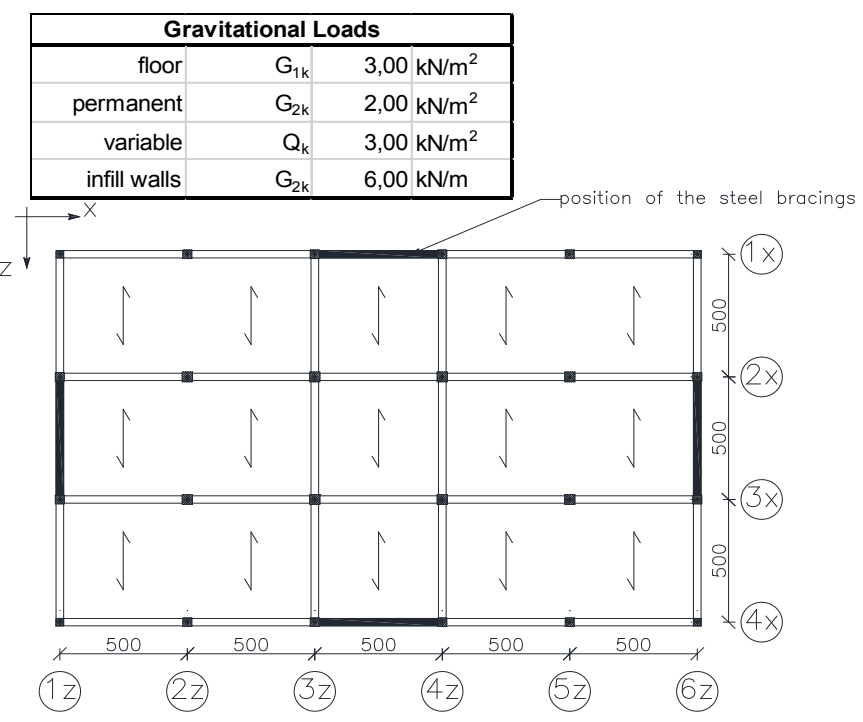

\begin{tabular}{|c|c|}
\hline \multicolumn{2}{|c|}{ Corner Column } \\
\hline section & $30 \times 30$ \\
\hline long. Reinf. & $8 Ф 12$ \\
\hline stirrups & $\Phi 8 / 25$ \\
\hline \multicolumn{2}{|c|}{ Border Column } \\
\hline section & $35 \times 35$ \\
\hline long. Reinf. & $12 \Phi 12$ \\
\hline stirrups & $\Phi 8 / 25$ \\
\hline \multicolumn{2}{|c|}{ Centre Column } \\
\hline section & $40 \times 40$ \\
\hline long. Reinf. & $12 \Phi 12$ \\
\hline stirrups & $\Phi 8 / 25$ \\
\hline \multicolumn{2}{|c|}{ Beam } \\
\hline section & $30 \times 50$ \\
\hline $6 Ф 12$ & sup \\
\hline 3Ф12 & inf \\
\hline \multicolumn{2}{|c|}{ at the ends } \\
\hline 3Ф12 & sup \\
\hline $6 Ф 12$ & inf \\
\hline \multicolumn{2}{|c|}{ in the middle } \\
\hline
\end{tabular}

Figure 9: Sample existing structure and position of the steel bracing equipped with SSS devices.

according to the codes and practices in force in Italy in 1960s and 1970s $[27,28]$, is considered herein as a case-study with the aim of investigating the influence of the accelerated low-cycle fatigue degradation exhibited by the SSS devices under consideration. Figure 9 shows its plan view and main member sections.

A cylindrical compressive strength $\mathrm{f}_{\mathrm{cm}}=16 \mathrm{MPa}$ was considered for concrete, while steel type FeB22k (medium tensile strength $f_{s m}=220$ 
MPa) was considered for rebars. HEA200 steel profiles made of S275 were considered to simulate the bracings supporting the dissipative devices. The frame was equipped with SSS devices for enhancing its seismic response: Figure 10 shows the front view of the RC structure with steel bracings and dissipative devices.

The effects of the low-cycle fatigue of the dissipative devices on the overall global response was investigated by performing Incremental Dynamic Analyses (IDA) on three different models of the RC structure under consideration:

- The first model represented the existing structure in its asbuilt configuration, without any steel bracing and device;

- In the second one steel bracings and dissipative devices were included, but the low-cycle fatigue phenomenon affecting SSS devices was not modelled;

- The third one considered the RC structures with both Y-shape steel bracings and SSS dissipative devices in which the effects of lowcycle fatigue are taken into account according the model outlined in the previous section.

Beams, columns and braces were modelled through distributed plasticity elements taking into account both geometric and mechanical non linearity. Ordinary stress-strain laws, namely Concrete01 and Steel01 [16], were adopted for materials.

Seven accelerograms were selected in the Pacific Earthquake Engineering Research Center (PEER) database and scaled in order to obtain a spectral acceleration $S_{2}\left(T_{1}\right)$ corresponding to the first vibrational mode of the analysed structure ranging between 0 and $5,00 \mathrm{~m} / \mathrm{s}^{2}$. Table 3 reports their main characteristics: PGA and PGV denote the Peak Ground Acceleration and the Peak Ground Velocity, respectively. Moreover, the damage index $I_{D}$ was evaluated according to the following equation [19]:

$$
I_{D}=\frac{\int_{0}^{t_{e}}[a(t)]^{2} d t}{\text { PGA } P G V}=\frac{I_{A}}{P G A \cdot P G V},
$$

in which $I_{A}$ is the so called Arias Index.

As it well known, the $I_{D}$ parameter is strictly related to the energy content of earthquakes. As it can be read in Table 3, the selected accelerograms are characterised by different $I_{D}$ values with the aim of investigating the influence of low-cycle fatigue on the structural response to seismic actions characterised by significantly different damage potential.

\section{Results of IDA analysis}

The results of IDA performed on the three different models are reported in terms of acceleration vs. top displacement $\left(S_{a}-\Delta_{\text {top }}\right)$ : particularly, Figure 11 reports the average (Figure 11a) and the maximum (Figure 11b) displacement demands obtained for the three series of incremental analyses proposed in this study. As expected, the use of steel bracings and dissipative SSS devices significantly reduces the top displacement demand on the structure: in fact, the demand determined for the existing structure is at least halved by the contribution of steel bracings.

However, a significant difference emerges between the results of analyses obtained by either considering or neglecting the effect of lowcycled fatigue in the dissipative devices. This difference is especially clear in terms of average value of $\Delta_{\text {top }}$ (Figure 11a) for medium-to-high seismic signals (i.s. $\mathrm{S}_{\mathrm{a}}>2.5 \mathrm{~m} / \mathrm{s}^{2}$ ).

Moreover, the significant difference emerging between the average values (Figure 11a) and the corresponding maximum ones (Figure 11b) suggests that the seismic response is affected by a significant record-byrecord variability.

Therefore, Figures 12-14 show the response obtained in the three series of analyses for each one of the seismic signals (dashed one) and the same average response already plotted in Figure 11a. As it is expected, this dispersion is higher for analyses implying higher levels of nonlinear response. Therefore, it is significant for the existing structure (Figure 12), whereas it appears to be strongly reduced for the retrofitted structures, if the low-cycled fatigue effect is neglected (Figure 13). Conversely, the record-to-record variability is once again pronounced if the low-cycle fatigue effect of SSS devices is duly taken into account.

A further investigation into the seismic response of the three analysed structures under each one of the seismic signals under consideration reveals that the role of low-cycle fatigue is particularly

\begin{tabular}{|l|l|c|c|c|}
\hline Earthquake & Station & PGA [g] & PGV [m/s] & I \\
\hline Mammoth Lakes & Long Valley Dam & 0.2388 & 0.1512 & 0.43 \\
\hline Kocaeli & Duzce & 0.2825 & 0.5206 & 1.52 \\
\hline Trinidad & Rio Dell Overpass & 0.4153 & 0.3915 & 0.52 \\
\hline Loma Pietra & Fremont & 0.1273 & 0.1105 & 1.08 \\
\hline Chalfant Valley-02 & Zack Brother Ranch & 0.3844 & 0.3891 & 0.79 \\
\hline San Fernando & LA Hollywood Stor & 0.1797 & 0.1760 & 0.80 \\
\hline Chi-Chi & TCU055 & 0.2461 & 0.3079 & 1.28 \\
\hline
\end{tabular}

Table 3: Characteristics of the accelerograms used in dynamic analysis.

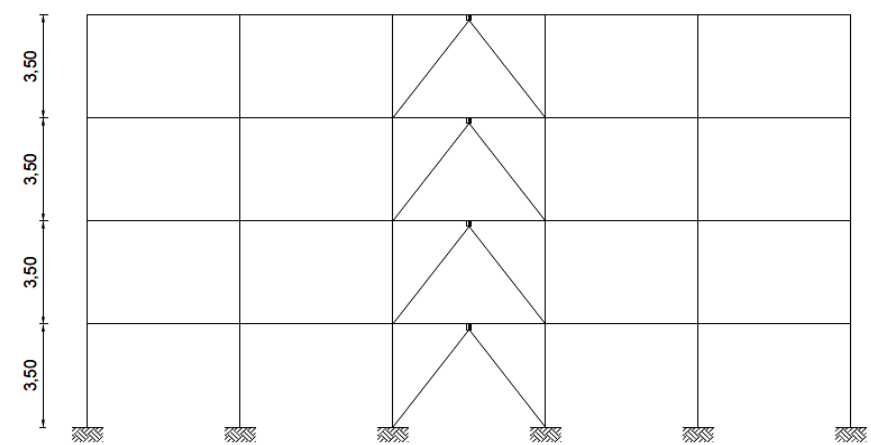

Figure 10: Perspective view of the structure and V-configuration of the steel bracings. 


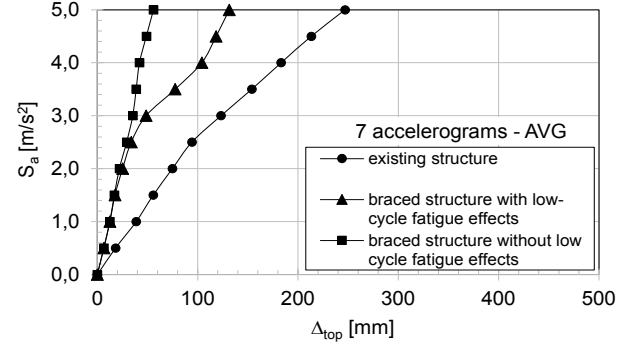

(a)

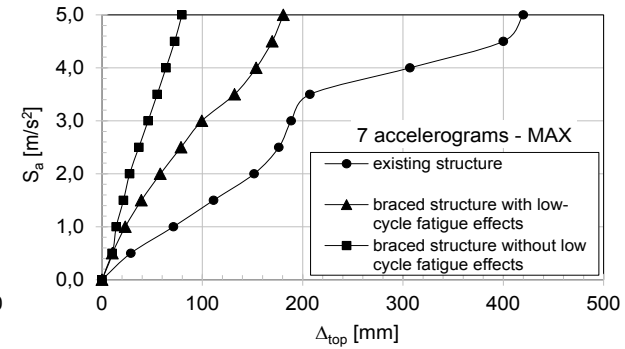

(b)

Figure 11: IDA curves: average (a) and maximum (b) of the 7 seven seismic signals.

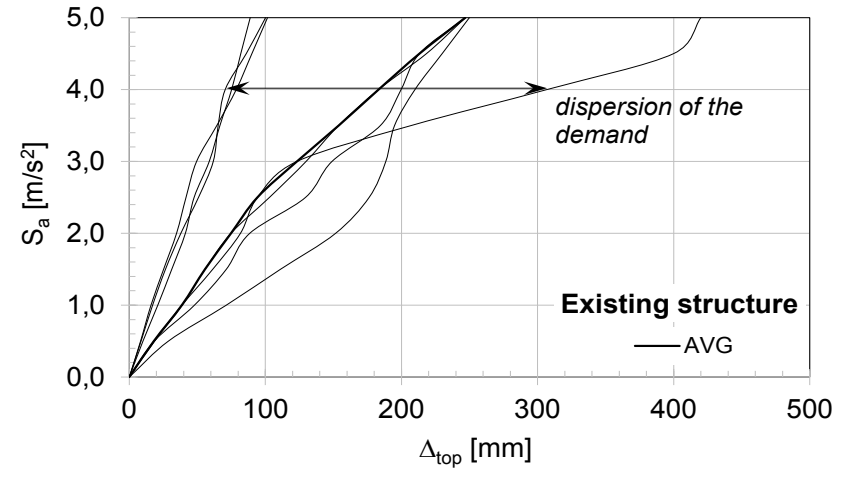

Figure 12: IDA curves: single signal and average (existing structures).

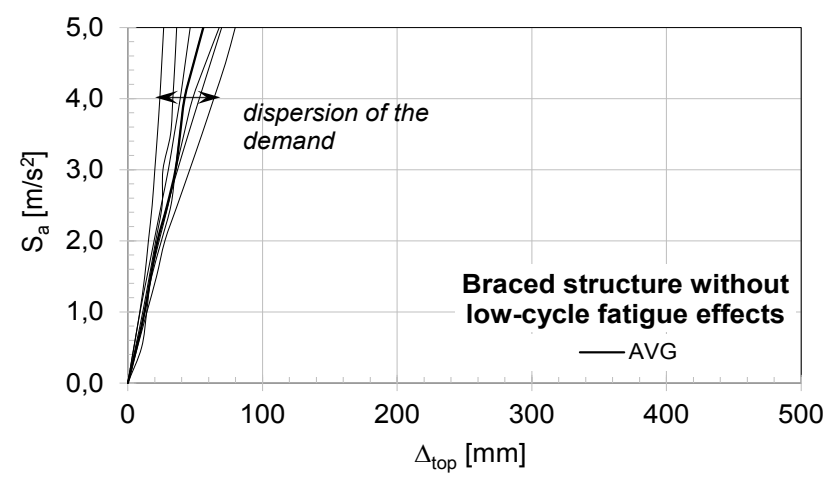

Figure 13: IDA curves: single signal and average (retrofitted structure - no low-cycle fatigue).

pronounced in certain cases. In particular, Figure 15 depicts the IDA curves of the two accelerograms characterised by the lower and the higher $I_{D}$ values [19], respectively: they also results, respectively, in the minimum and maximum scatter between the two IDA curves representing the seismic response of the braced structure determined by either considering or neglecting the low cycle fatigue phenomenon on the SSS devices.

Similarly, Figure 16 shows the horizontal force-displacement response of a device located at the first storey of the structure under consideration. Particularly, it depicts the behaviour obtained by performing the nonlinear dynamic analyses for the same couple of accelerograms characterised by the lower and the higher $\mathrm{I}_{\mathrm{D}}$ value. The

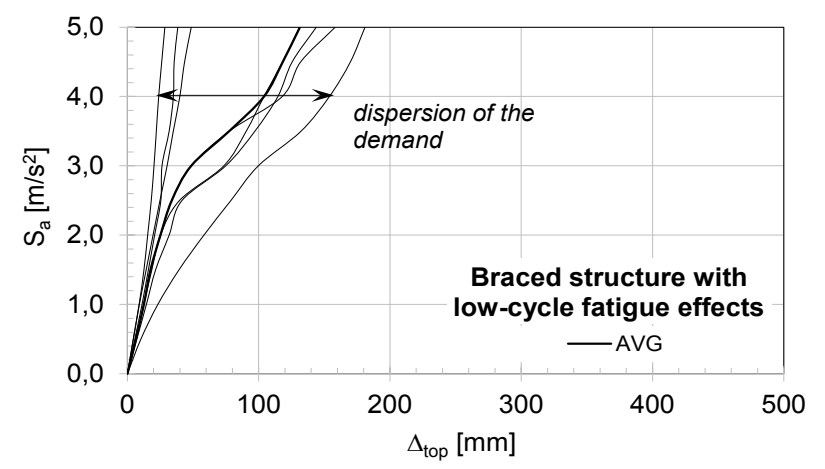

Figure 14: IDA curves: single signal and average (retrofitted structure - lowcycle fatigue).

results confirm that the accelerogram with higher $I_{D}$ value leads to a faster degradation of the SSS device response.

Therefore, the results reported in Figures 15 and 16 highlight that the influence of the low-cycle fatigue may be correlated with the value of $I_{D}$. This conjecture can be assessed by analysing the ratio between the top displacement of the structure determined by considering the effect of low-cycle fatigue and the corresponding one determined by neglecting that phenomenon: it is clear that if this ratio is close to one, the low-cycle fatigue effect is negligible, whereas high values of the same ratio testifies that displacement demand is influenced by the aforementioned phenomenon. The $\Delta_{\text {top,withLCF }} / \Delta_{\text {top,withoutLCF }}$ is reported in Figure 17 for each accelerogram with respect to the corresponding $I_{D}$ value: a close correlation emerges between the two quantities and, hence, the above conjecture about the role of $I_{D}$ in controlling the influence of low-cycle fatigue on the global response of the retrofitted structure is confirmed.

Conversely, no correlation can be recognised between the displacement demand ratio defined above and other relevant intensity measures of the seismic signals considered in the performed IDA (Figure 18).

\section{Conclusion}

The present paper is a contribution to understanding the consequences of the low-cycle fatigue degradation affecting some cost-competitive devices, such as the so-called Steel Slit Shape ones considered in this study. Particularly, the following observation deserves to be remarked:

- A numerical model based on using 1D frame-like finite 
Citation: Lima C, Martinelli E, Faella C (2015) Cost-competitive Steel Devices for Seismic Retrofitting of RC Frames: Model Identification and Nonlinear Analysis. J Steel Struct Constr 1: 104. doi:10.4172/2472-0437.1000104

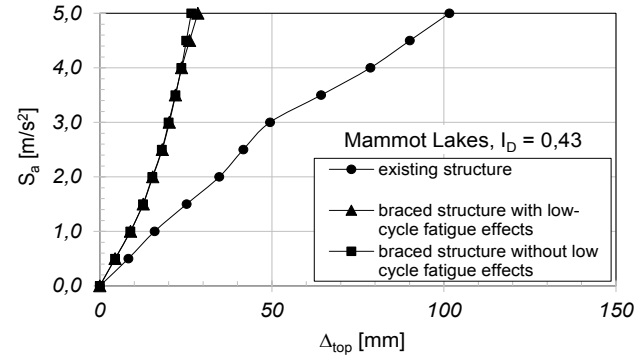

(a)

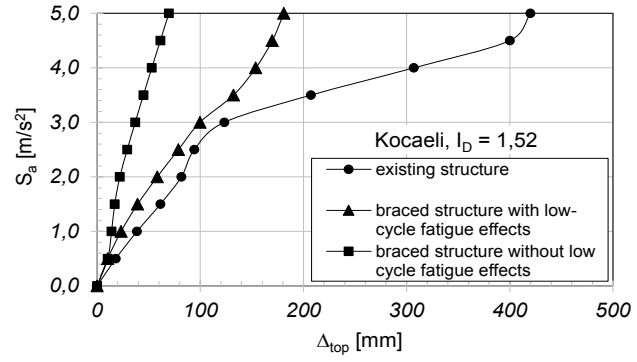

(b)

Figure 15: IDA curves of the Mammoth Lakes (a) and Kocaeliaccelerograms (b).
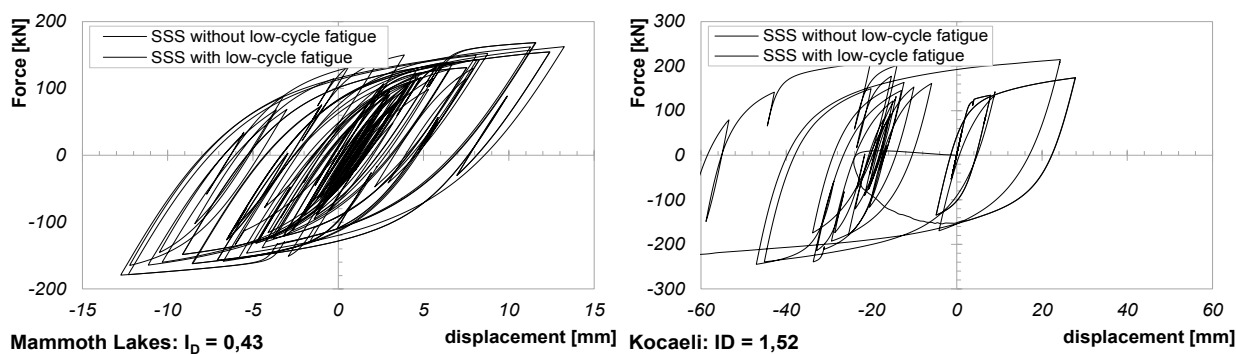

Figure 16: Cyclic response of a SSS device located at the first storey of the structure.

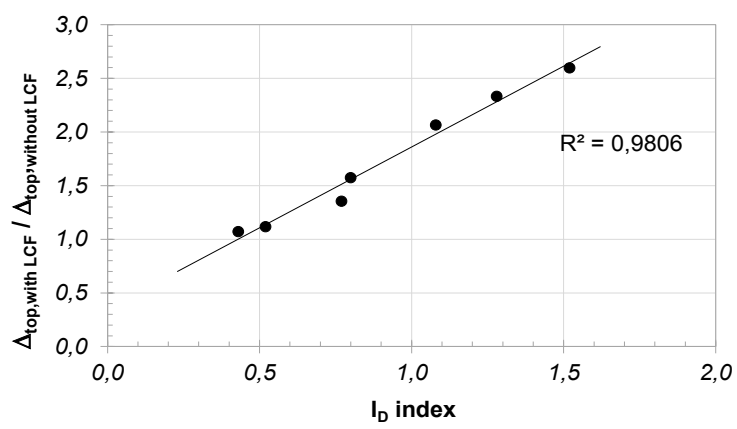

Figure 17: Correlation between the top displacement ratio and the ID index of each signal.
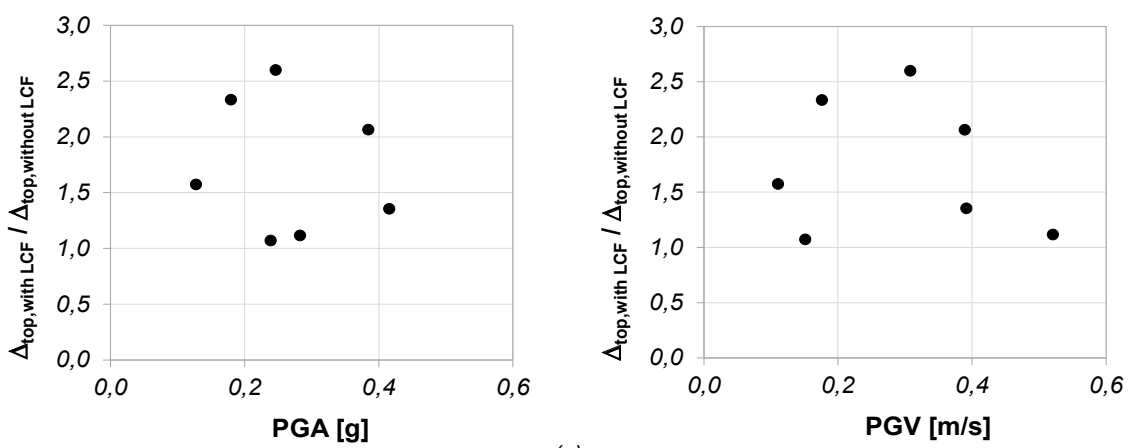

Figure 18: Correlation between the top displacement ratio and PGA (a) and PGV (b) of each signal. 
Citation: Lima C, Martinelli E, Faella C (2015) Cost-competitive Steel Devices for Seismic Retrofitting of RC Frames: Model Identification and Nonlinear Analysis. J Steel Struct Constr 1: 104. doi:10.4172/2472-0437.1000104

element and fiber section discretisation, generally available in designoriented codes for structural analysis was proposed for simulating the cyclic behaviour of a cost-competitive solution for realising dissipative devices, particularly suited for being used in retrofitting RC frames;

- Low-cycle fatigue degradation observed in experimental results was simply taken into account in the aforementioned model which was included in the global FE model of the retrofitted RC structure considered;

- The performed IDA demonstrates that the action of steel bracings and dissipative SSS devices significantly reduces the top displacement demand on the structure, but the low-cycle fatigue affecting the SSS devices generally influences the response of the retrofitted structure.

- In particular, low-cycle fatigue of SSS devices plays a role as high as the $I_{D}$ index of the seismic signal under consideration: among the other things, this observation suggests a possible criterion for selecting natural seismic signals to be employed in the structural analyses needed in practical retrofitting intervention.

Therefore, the results presented in this study have relevance for several issues dealing with the seismic retrofitting of existing RC frames: particularly, on the one hand, it demonstrates the potential of a cost-competitive dissipative device in retrofitting existing RC frames and, on the other hand, it shed a new light on the consequences of their more pronounced low-cycle fatigue degradation on the global seismic response of the retrofitted structure.

\section{Acknowledgement}

This study is part of the DPC-ReLUIS 2014-2018 Research Project whose financial support is gratefully acknowledged.

\section{References}

1. Kawashima K, Aydan O, Aoki T, Kisimoto I, Konagai K, et al. (2010) Reconnaissance investigation on the damage of The 2009 L'Aquila central Italy earthquake. Journal of Earthquake Engineering 14: 816-841.

2. CEN (2005) Eurocode 8, Design of structures for earthquake resistance. Part1: General rules, seismic actions and rules for building, EN 1998-1-1, European Committee for Standardization, Brussels (BE).

3. Faella C, Martinelli E, Nigro E (2008) A rational strategy for seismic retrofitting of rc existing buildings. Proceedings of the $14^{\text {th }}$ World Conference on Earthquake Engineering, Beijing, China

4. Mele M, Paciello SO, Perri F (2009) Hysteretic devices for seismic retrofitting of existing buildings. Innovative Strategies for structural Protection of Built Heritage- PRIN 2005, Polimetrica Publisher s.a.s., Monza (Italy).

5. Black CJ, Makris N, Aiken ID (2004) Coponent Testing, Seismic Evaluation and Characterization of Buckling. Restrained Braces J Struct Eng 130: 880-894.

6. Whittaker AS, Bertero VV, Thompson CL, Alonso LJ (1991) Seismic testing of steel plate energy dissipation devices. Earthquake Spectra 7: 563-604.

7. Tsai K, Chen H, Hong C, Su Y (1993) Design of steel triangular plate energy absorbers for seismic-resistant construction. Earthquake Spectra 9: 505-28.

8. Chan R-WK, Albermani F (2008) Experimental study of steel slit damper for passive energy dissipation. Engineering Structures 30: 1058-66.

9. Ghabraie K, Chan RWK, Huang X, Xie YM (2010) Shape optimization of metallic yielding devices for passive mitigation of seismic energy. Engineering Structures 32: 2258-67.

10. Benavent-Climent A, Morillas L, Vico JM (2011) A study on using wideflange section web under out-of-plane flexure for passive energy dissipation. Earthquake Engineering and Structural Dynamics 40: 473-90.
11. Nakashima M (1995) Strain-hardening behavior of shear panels made of lowyield steel. J Struct Eng ASCE 121: 1742-1749.

12. Nakashima M, Akazawa T, Tsuji B (1995) Strain-hardening behavior of shear panels made of low-yield steel. II: model J Struct Eng ASCE 121: 1750-1757.

13. Benavent Climent A, Oh SH, Akiyama H. Ultimate energy absorption capacity of slit-type steel plates subjected to shear deformations. J Struct Constr Eng 503: $139-145$

14. Faella C, Lima C, Martinelli E, Realfonzo R (2014) Steel bracing configurations for seismic retrofitting of a reinforced concrete frame. Structures and Buildings 167: 54-65.

15. Perri F, Faella C, Martinelli E (2013) Experimental tests on the cyclic response of common steel work hysteretic devices. XXIV Congresso CTA - Le Giornate Italiane della Costruzione in Acciaio, Torino, 30 settembe - 2 ottobre 2: 859-866

16. Mazzoni S, McKenna F, Scott MH, Fenves GL (2006) Open System for Earthquake Engineering Simulation, User Command-Language Manual. Pacific Earthquake Engineering Research Center, University of California, Berkeley.

17. Lima C, Martinelli E, Faella C (2015) Simulation of the low-cycle fatigue response of dissipative steel devices in retrofitted RC frames. OpenSEES Days, $2^{\text {nd }}$ Italian Conference, Salerno, Italy.

18. Assareh MA, Asgarian B (2008) Nonlinear behavior of single piles in jacket type offshore platforms using incremental dynamic analysis. American Journal of Applied Sciences 5: 1793-1803.

19. Cosenza E, Manfredi G (1997) The improvement of the seismic-resistant design for existing and new structures using damage criteria. In Seismic Design Methodologies for the Next Generation of Codes, Fajfar P, Krawinkler H. (eds). Balkema: Rotterdam.

20. Italian Ministry of Infrastructures and Transports (2008) Norme Tecniche per le Costruzioni (NTC 2008). D.M. 14/01/2008, Gazzetta Ufficiale n. 29 del 4 febbraio 2008 - Suppl. Ordinario n. 30 (in Italian).

21. Chan RWK, Albermani F, Williams MS (2009) Evaluation of yielding shea panel device for passive energy dissipation. Journal of Costructional Steel Research 65: 260-268

22. ECCS (1986) Technical Committee 1 - Structural Safety and Loadings Technical Working Group 1.3 - Seismic Design, Recommended Testing Procedure for Assessing the Behaviour of Structural Steel Elements under Cyclic Loads, ECCS publication.

23. Plamgreen A (1924) Die Lebensdauer von Kugellagern. Verfahrenstechinik, Berlin, German.

24. Miner MA (1945) Cumulative damage in fatigue. Journal of Applied Mechanics 67: 159-164.

25. Chan RWK, Albermani F (2008) Experimental study of steel slit damper for passive energy dissipation. Engineering Structures 30: 1058-1066.

26. Ballio G, Castiglioni CA (1995) A Unified Approach for the Design of Stee Structures under Low and/or High Cycle Fatigue. Journal of Constructional Steel Research.

27. Regio Decreto (1939) Norme per la esecuzione delle opera in conglomerate cementizio semplice ed armato.

28. Santarella L (1966) Prontuario del Cemento Armato. XXV Edizione, Hoepli, Milano. 